\title{
Graduación de sanciones en la responsabilidad del abogado por las faltas cometidas por personas no-abogadas que se encuentran bajo su supervisión"
}

\author{
GLORIA ZUBIZARRETA RONDÓN
}

SUMARIO: I. INTRODUCCIÓN: BREVE PANORAMA DE LA PROFESIÓN Y PRE-
SENTACIÓN DEL TEMA.- II. ¿ES IMPORTANTE EL TEMA A SER ANALIZADO?
ANÁLISIS A LA AFECTACIÓN DE VALORES EN EL SISTEMA DE JUSTI-
CIA.- III. SUPUESTOS DE IMPUTACIÓN.- IV. GRADUACIÓN DE SANCIONES.

\section{INTRODUCCIÓN: BREVE PANORAMA DE LA PROFESIÓN Y PRESENTACIÓN DEL TEMA}

La abogacía actualmente está experimentando una serie de cambios que han ocasionado transformaciones en el ejercicio del Derecho, desde fenómenos causados por el desarrollo del mercado - como la competencia intensa entre estudios de abogados, la especialización del abogado en un campo específico y demás-, hasta la forma como la sociedad percibe la figura del abogado. En este contexto, y desde hace ya algún tiempo, han surgido códigos de ética que se orientan cada vez más a regular deberes específicos del abogado, no solo en relación con la profesión, sino también respecto de los distintos actores que se ven involucrados en ella.

Así, desde una visión antigua, en la que la abogacía se concebía como un ministerio, ${ }^{1}$ el abogado era aquel defensor de la justicia que practicaba el Derecho por un sentimiento de altruismo, y la posibilidad de publicitar sus servicios o concebir los conocimientos legales como medio para obtener lucro era impensable.

Gradualmente, con la democratización de los centros de estudios universitarios, más personas tuvieron acceso al estudio de la profesión, lo que causó una masificación en el mercado en lo concerniente a la oferta de servicios legales. De este modo, los abogados comenzaron a competir por el público mediante diferentes medios — como la publicidad- y principalmente buscaron optimizar los servicios legales ofrecidos con los mayores estándares de calidad posible, con lo cual brindaron las mejores garantías a ssu clientes en las relaciones abogado-cliente establecidas.

Por otro lado, la base que permite que el sistema de justicia y que la profesión de la abogacía funcione es la confianza mutua que existe entre el cliente y el abogado respecto del manejo del asunto encomendado. 
El cliente no solo confía en el manejo diligente del abogado respecto de su problema, sino también en el cuidado que tendrá con la información proporcionada y, en general, con todo lo que alcance al patrocinio. Por eso, el abogado tiene para con el cliente la obligación de realizar un patrocinio debido, que conlleva una serie de deberes y obligaciones, tales como diligencia, competencia, lealtad y veracidad, ${ }^{2}$ entre otros.

Actualmente, la oferta de servicios legales se configura sobre todo en estudios jurídicos que compiten arduamente entre ellos y que han ido creciendo estructuralmente durante los últimos diez años, debido al grado de inversión privada y consecuente desarrollo nacional que ha generado un gran crecimiento económico en diversos sectores. En consecuencia, existe una importante presencia de profesionales o personas no-abogadas que trabajan dentro de ellos realizando labores de diversa índole, esenciales en muchos casos para la existencia diaria de tales estudios jurídicos. Debido a que estas personas se involucran en mayor medida con temas directamente relacionados con el patrocinio que tiene el abogado con un cliente, es necesario regular también su conducta, puesto que esta afecta directamente los intereses de los clientes, aun así estos no se den cuenta del rol que cumplen los no-abogados en sus asuntos. Imaginémonos, por ejemplo, un proceso judicial llevado por el abogado X por encargo del cliente $\mathrm{Y}$. El día en que se vence el plazo para presentar el recurso de apelación correspondiente, el abogado entrega el documento a uno de los procuradores del estudio. El procurador, por descuido y por la carga de trabajo que tiene — debe, además, presentar diversos escritos en otros juzgados por encargo de otros abogados del mismo estudio-, olvida presentar la apelación correspondiente, con lo que perjudica al cliente. En este caso, cabría preguntarse: ila culpa es solo del procurador? ¿Tiene el abogado X, quien lleva el caso directamente, alguna responsabilidad por el olvido del procurador? iA quién se debe sancionar por esta falta cometida: al abogado, al procurador o a ambos?

El propósito del presente ensayo es establecer una graduación de sanciones adecuada al abogado en los casos en que las personas no-abogadas que trabajan bajo su supervisión cometan faltas profesionales $-\mathrm{O}$, en todo caso, faltas propias de su oficio- Para ello, nos concentraremos básicamente en tres fuentes, que servirán para extraer diversos supuestos que configurarán la graduación de sanciones. Estas tres fuentes están compuestas por la doctrina, la jurisprudencia y por las normas del Derecho comparado.

Las fuentes, como se verá más adelante, tienen origen extranjero, ya que en Perú, además de no haberse desarrollado este tema de trascendental importancia, aún se tiene, con el actual Código de Ética, la visión antigua de la profesión, que está desfasada y que, lamentablemente, no contiene una norma con el supuesto mencionado. Ello permite que hoy en día sigan registrándose conductas indebidas por personas no-abogadas

2 Estos deberes se encuentran actualmente recogidos en el artículo 8 del Proyecto de Código de Ética y Responsabilidad del Profesional en Derecho, de junio de 2008. 
en el marco del patrocinio - como es el caso de las famosas «coimas», las cuales configuran un gran daño para la profesión-.

\section{II. ¿ES IMPORTANTE EL TEMA A SER ANALIZADO? ANÁLISIS A LA AFECTACIÓN DE VALORES EN EL SISTEMA DE JUSTICIA}

Hasta este momento, el lector puede considerar que, aunque el tema presentado es novedoso y en cierto modo controversial, no es tan importante como las discusiones existentes sobre la oportunidad de revelación del secreto profesional, los límites a la publicidad del abogado o los diversos tipos de conflictos de intereses, que podrían enriquecer más la práctica ética del medio profesional, ya que son cuestiones que afectan directamente la imagen y el ejercicio de la profesión. Pues bien, este capítulo se centra principalmente en el estudio de los valores que se vulneran cuando una persona no-abogada comete faltas en el ejercicio de su profesión u oficio y en los alcances de la responsabilidad del abogado, los cuales pueden afectar, tanto como las otras discusiones, la existencia de un adecuado desarrollo del sistema de justicia y de la visión que significa la profesión del abogado en el Perú.

En primer lugar, es preciso recordar que el abogado, actualmente, es visto como aquella persona que persigue solo sus propios intereses económicos, que lucra con problemas ajenos y que es en extremo inescrupuloso al buscar resultados favorables para sus clientes mediante medios extralegales - como la influencia indebida en autoridades y los sobornos, por mencionar algunos- Como consecuencia de esta pésima visión de la profesión, la confianza en el sistema de justicia por parte de la población -y por algunos profesionales abogados también— es mínima.

En el caso planteado, podemos apreciar que se vulneran, con las faltas de las personas no-abogadas supervisadas por un abogado, dos esferas centrales en la práctica del derecho.

En primer lugar, se encuentra la relación abogado-cliente, en tanto que se afecta la confianza que tiene este último en el abogado y en la forma en que el letrado va a manejar el asunto encomendado, vinculada íntimamente con la cuestión del patrocinio debido.

«Confianza», según la Real Academia de la Lengua Española es una «esperanza firme que se tiene de alguien o algo»; relacionada con el comercio, es aquel «pacto o convenio hecho oculta y reservadamente entre dos o más personas». ${ }^{3}$ En ese sentido, el cliente debería sentir que puede contarle al abogado absolutamente todo lo relacionado con el asunto encargado, en la medida en que ello contribuirá a que el profesional desarrolle una estrategia adecuada de defensa para dirigir al

3 Página web de la Real Academia de la Lengua Española. <www.rae.es>. Fecha de consulta: 15 de noviembre de 2008, 12:21 p.m.

GRADUACIÓN

DE SANCIONES

EN LA RESPON-

SABILIDAD DEL

ABOGADO POR

LAS FALTAS

COMETIDAS POR

PERSONAS NO-

ABOGADAS QUE

SE ENCUENTRAN

BAJO SU SUPER-

VISIÓN 
cliente hacia la solución más beneficiosa del caso. Si existen dudas acerca de las personas que trabajan cerca del abogado - por ejemplo, riesgo de que los datos exclusivamente conocidos por el abogado y los que trabajan con él se filtren a la prensa, como ocurre con las fuentes anónimas internas-, el cliente se abstendrá de exponer algunos datos relevantes para el caso, lo cual afectará su derecho de defensa y, en última instancia, el Estado de derecho mismo, al no funcionar adecuadamente las garantías fundamentales por la desconfianza generalizada a los operadores del Derecho.

Respecto de la esfera de la relación abogado-cliente, encontramos que el riesgo con las faltas de las personas no-abogados se refiere principalmente al tema del patrocinio debido del abogado. El patrocinio debido es aquella obligación que el abogado tiene de llevar el asunto de su cliente de manera diligente, al utilizar todos los medios legales para ello, y con un estándar de competencia adecuado. En el Proyecto de Código de Ética, encontramos que el artículo 24 consagra los deberes de diligencia y competencia. ${ }^{4}$

En el caso concreto, encontramos que las faltas mencionadas afectan principalmente el deber de diligencia y, por ende, la manera negligente y deficiente en la que el abogado lleva el caso encomendado.

La diligencia es la actuación con «prontitud, dedicación y empeño» ${ }^{5}$ que debe realizar el abogado para proteger los intereses del cliente. Este deber nace con el surgimiento de la relación abogado-cliente y se extingue cuando esta última también lo hace. Se refiere al cuidado y manejo de los asuntos confiados al abogado, vigilancia y dedicación de este, y subsiste aun así el abogado haya delegado el caso a otro profesional a su cargo — sea delegación total o parcial-.${ }^{6}$ Es decir, el abogado debe cuidar en todo momento, dentro de un parámetro de racionabilidad, que las actuaciones de las personas a su cargo sean correctas y que obedezcan al interés del cliente. Las faltas que cometa supondrán un perjuicio para el interés del cliente dentro del patrocinio y reflejarán también el descuido del abogado en el asunto encomendado. Así, se devengará la responsabilidad del abogado frente al cliente, ya que este contrató específicamente al abogado para que lo patrocine de manera adecuada, dentro de lo cual se incluye la supervisión sobre el comportamiento de sus subordinados.

La segunda esfera afectada es la de apariencia debida de la profesión, referida a la imagen de la figura del abogado en el entorno social. En la medida en que el abogado tiene un deber de supervisión sobre las

4 Artículo 24. Competencia y diligencia profesional. «Es deber del abogado defender el interés del cliente de manera diligente y con un elevado estándar de competencia profesional. Por ello, el abogado debe mantenerse actualizado en el conocimiento del Derecho, principalmente en el área de su especialidad, a través de una formación continua».

5 Boza Dıвós, Beatriz y Christian Chocano. Ámbitos de la responsabilidad en el ejercicio. CD Material del Curso de Ética y Responsabilidad Profesional, p. 3.

6 Confróntese Paredes Marroquí, Juan Alberto. «Alcances del deber de diligencia en la relación abogadocliente». Revista Derecho y Sociedad, n²4, Lima, 2006, pp. 369-377. 
personas que trabajan para él, se presume que conoce sus acciones. Si las faltas por parte de las personas supervisadas fueran reiteradas, podría suponerse que el abogado estaría no solo apañando conductas indebidas, sino incluso que estaría en favor de ellas o, más grave aún, que participa directamente en su concreción. Situaciones como esta, que ocurren a menudo, consolidan la visión del abogado corrupto que ya comentamos y generan desconfianza generalizada en la población. Se siente que se carece de cuidado en el manejo de los casos y que el abogado no los considera importantes, ya que no les presta la suficiente atención. Todo ello posibilita, así, las faltas de las personas que laboran bajo el cargo del abogado.

\section{SUPUESTOS DE IMPUTACIÓN}

A continuación, expondremos los supuestos de imputación existentes en las normas de Derecho comparado, doctrina y jurisprudencia.

\section{Normas de Derecho comparado}

El vigente Código de Ética de 1997 no regula la responsabilidad del abogado por faltas cometidas por las personas que se encuentran bajo su supervisión. Hemos recurrido a las normas internacionales de ética sobre la materia a fin de identificar los límites de la responsabilidad del abogado y los deberes que se le imponen en este contexto.

Una primera fuente sobre la materia es el Código Deontológico de la Comunidad Europea, en cuyo artículo 2.3.4 expresamente señala el deber del abogado de cuidar que el secreto profesional de su cliente sea respetado tanto por su personal como por cualquier persona que colabore con este en el manejo del asunto. ${ }^{7} \mathrm{El}$ secreto profesional es una institución básica para la existencia del Derecho, ya que permite que el cliente, como mencionamos anteriormente, ejercite de forma efectiva su derecho a obtener la mejor defensa posible. Por ello, es de vital importancia que toda la información conocida por los abogados sea cuidadosamente resguardada. Este deber se extiende a las personas que conozcan el asunto directa o indirectamente. En tal sentido, por el mismo hecho de ser parte de la organización del estudio jurídico o de la consulta del abogado, toda persona no-abogada que se encuentre bajo su supervisión debe respetar la información confidencial proveída por el cliente. El incumplimiento de este deber acarrea también la responsabilidad del abogado, ya que estaría faltando al deber de vigilancia debida, que analizaremos como criterio en el último capítulo del presente ensayo.

El Model Rules of Professional Conduct ABA, de los Estados Unidos de Norteamérica, brinda más luces al respecto: en el rule $5.3,{ }^{8}$ que se refiere

7 Artículo 2.3.4. «El Abogado debe hacer respetar el secreto profesional por su personal y por cualquier persona que colabore con él en su actividad profesional».

8 «With respect to a nonlawyer employed or retained by or associated with a lawyer:

(a)apartner,andalawyerwhoindividually ortogetherwithotherlawyerspossessescomparablemanagerial authority in a law firm shall make reasonable efforts to ensure that the firm has in effect measures giving reasonableassurancethattheperson'sconductiscompatiblewiththeprofessionalobligationsofthelawyer;

GRADUACIÓN

DE SANCIONES

EN LA RESPON-

SABILIDAD DEL

ABOGADO POR

LAS FALTAS

COMETIDAS POR PERSONAS NO-

ABOGADAS QUE

SE ENCUENTRAN

BAJO SU SUPER-

VISIÓN 
a la responsabilidad de las personas no-abogadas que trabajan con los abogados, se incluyen tres supuestos concretos:

1. Que el socio o el abogado que tiene capacidad de manejo en la firma realice un esfuerzo razonable para que se instauren efectivamente mecanismos que aseguren que el comportamiento de la persona no-abogada —empleado, consultor o asociado- es compatible con las obligaciones profesionales del abogado. En ese sentido, implica una voluntad firme de instaurar políticas éticas dentro del centro de trabajo y que efectivamente estas sean cumplidas por el personal. De tal modo, se puede garantizar, en cierta medida, una conducta debida que no impacte negativamente en el manejo de asuntos del cliente. Sin embargo, mientras implique voluntad y esfuerzo, no es suficiente para asegurar que estas medidas se concreten, máxime si muchas firmas de abogados ven que instaurar estos mecanismos puede resultar costoso - por el monitoreo constante y el análisis de efectividad de las medidas, entre otras evaluaciones continuas que deben ser realizadas-.

2. Que el abogado que tiene directa autoridad de supervisión sobre la persona no-abogada realice esfuerzos razonables para asegurar que la conducta del supervisado sea compatible con sus deberes profesionales. Como podemos apreciar, es muy similar al primer supuesto, en cuanto encierra la voluntad y los esfuerzos del abogado, si bien especifica a la persona a la que se le impone. En ese sentido, el abogado encontraría muy fácil eludir este deber, en cuanto que no encierra medidas concretas que pudieran exigírseles, ya que se limita a un «esfuerzo razonable», un parámetro muy subjetivo.

3. El tercer supuesto se subdivide en dos postulados, que consideramos regulan de mejor manera la responsabilidad del abogado. El primero de ellos se refiere a la responsabilidad del abogado por la conducta de la persona bajo su supervisión, si es que el primero ordena la comisión de la conducta que genera la falta o si, sabiendo del comportamiento indebido, la ratifica. En este caso se regula la relación directa del abogado con la conducta indebida, que configura un supuesto grave de responsabilidad, ya que no solo busca evadir su responsabilidad al recurrir a otra persona, sino que está proyectando una imagen indebida de la profesión, puesto que ratifica la comisión de una actuación que atenta contra la ética profesional al alentar — mediante la ratificación_ — la 
continuidad de este tipo de faltas que le proporcionan un beneficio propio. Otra vez, estaríamos frente a la imagen del abogado que utiliza cualquier medio para ganar su caso, a costa de actos indebidos.

El segundo postulado se refiere al conocimiento que podría tener el socio o abogado con mando gerencial o aquel abogado que tenga deber de supervisión directa y que, conociendo la infracción cuando sus consecuencias pudieran ser evitadas o mitigadas, no toma acción alguna en el caso. En este supuesto, se sanciona al abogado con responsabilidad porque, a pesar de haber podido realizar medidas que impidan la concreción de perjuicios para algún tercero —o para el mismo cliente, de ser el caso-, no lo hizo, aun cuando ello se encontraba dentro de su esfera de acción. La pasividad del abogado es reprochable porque busca no arriesgarse personalmente y permite que los efectos de la falta recaigan negativamente sobre alguien ajeno a la organización.

Como hemos podido apreciar, estos códigos o reglas de conducta para los abogados han recogido ciertos supuestos sobre la responsabilidad del profesional en Derecho respecto de las personas no-abogadas que supervisan. Hay mención directa sobre el deber de vigilancia del abogado y de la correcta supervisión de las personas en el centro de trabajo; en realidad, la responsabilidad del abogado es importante, ya que parte de la profesión es ser un ejemplo para aquellos que lo rodean. Al demostrar que no tolera las faltas éticas de las personas no-abogadas que trabajan con él, está instaurando un ejemplo a seguir en el interior de su organización. Por el contrario, si no se molesta en sancionar las faltas, proyecta una posición de compartir comportamientos indebidos, que a la larga será perjudicial no solo para el adecuado manejo de los asuntos que tiene a su cargo, sino para la reputación de la firma a la que pertenece.

\section{Doctrina}

La doctrina que hemos podido encontrar se centra específicamente en las personas no-abogadas que trabajan para firmas de abogados o que colaboran con abogados que forman parte de ellas. Sin embargo, por la naturaleza del asunto, es también aplicable a otras situaciones en donde los abogados trabajan con personas no-abogadas, como órganos gubernamentales o la fiscalía de cada nación.

Un primer supuesto de imputación que encontramos desarrollado por la doctrina es aquel en que el abogado es responsable por la conducta de los investigadores que contrata para obtener información en el asunto de un cliente. Tal como Barry T. Temkin expone en su ensayo «Deception in Undercover Investigations: Conduct Based vs. Status Based Ethical Analysis», ${ }^{9}$ se pueden presentar varias situaciones que comprometen

9 Temkin, Barry R. «Deception in Undercover Investigations: Conduct Based vs. Status Based Ethical Analysis». Seattle University Law Review, vol. 32, no 1, 2008, pp. 123-175.. Base de datos Westlaw.

GRADUACIÓN

DE SANCIONES

EN LA RESPON-

SABILIDAD DEL

ABOGADO POR

LAS FALTAS

COMETIDAS POR PERSONAS NO-

abOgadAs Que SE ENCUENTRAN BAJO SU SUPERVISIÓN 
éticamente al abogado por las actuaciones del investigador que colabora con él. Así, se discute si es permitido que un investigador se acerque a la contraparte para sonsacarle información y que falsee su identidad para conseguir que esta admita hechos o imputaciones, ya que existe una prohibición en la mayoría de códigos sobre la comunicación del abogado con la contraparte, sin la presencia del abogado que la representa. En la medida en que el investigador actúa por órdenes directas del abogado que lo contrató, y considerándose que la relación con la contraparte es indirecta - por la intermediación del investigador-, muchas cortes de los Estados Unidos han considerado que esto calificaría como deshonestidad y fraude, y lo proscriben terminantemente respecto del abogado y del investigador. Con ello se responsabiliza éticamente al primero por la actuación del segundo, ya que se considera que cometen actos indebidos que atentarían contra el derecho de defensa de la contraparte y que utilizan medios indebidos —engaño, principalmente- para obtener pruebas, lo cual puede suponer grave daño y lesión — por los efectos de la recolección de evidencia-.

Una gran excepción que se acepta en la mayoría de cortes de los Estados Unidos es el caso de las investigaciones encubiertas gubernamentales, en las que no se sanciona al abogado a cargo de la investigación —que tendrá a diversos agentes policiales bajo su mando y responsabilidad-. Por la naturaleza de las investigaciones, ya que se protegen valores públicos — seguridad nacional, convivencia pacífica de los ciudadanos, entre otros- , se acepta que los agentes infiltrados recurran al fraude y al engaño para obtener pruebas que pudieran demostrar la culpabilidad del acusado en un juicio posterior. Sin embargo, no se establece siquiera la posibilidad de que un abogado defensor pudiera hacer lo mismo para salvaguardar el derecho de su cliente o, en todo caso, para obtener los medios que pudieran atenuar la pena para su defendido.

Es interesante analizar, sin embargo, lo que sucede en los casos de litigios por propiedad intelectual. Ya que sin la intervención del investigador no se puede recolectar evidencia de la vulneración de derechos de propiedad - sobre una marca registrada- y que, si el investigador se descubre como agente de la compañía que tiene los derechos correspondientes es casi certero que no obtendrá evidencia de la infracción de una conducta, diversas cortes aceptan que se utilice el engaño para recolectar la evidencia necesaria para armar el caso. Sin embargo, el límite para el engaño sería el propósito válido del abogado —a través del cual actúa el cliente- - y el ocultamiento de identidad del investigador, y con ello se justificaría que sería casi imposible conseguir las pruebas de la vulneración de la propiedad intelectual por otros medios. ${ }^{10}$

Otro supuesto de imputación que podemos encontrar en doctrina se refiere a los estudiantes de Derecho que realizan prácticas en las 
firmas de abogados. La fuente ${ }^{11}$ que consultamos proviene de los Estados Unidos. Recordemos que las prácticas (externships) en aquel país están reguladas de manera clara y que los abogados que tienen alumnos a su cargo poseen una serie de deberes para con sus alumnos —entre los que se cuentan enseñanza, orientación ética y ejemplo a seguir-. Las prácticas también están controladas por la figura del supervisor de la facultad del practicante, quien se asegurará de que aquellas se desenvuelvan de forma correcta.

El primer supuesto que encontramos es el caso de conflicto de intereses de los practicantes en el sitio en el que actualmente realiza su pasantía. Debido a que el alumno ha podido realizar prácticas varias veces, es probable que en la actual firma en la que se desempeña se le adjudique el caso de un cliente - bajo la supervisión del abogado encargado, por supuesto- y se dé cuenta de que, por una práctica pasada, tiene acceso a información privilegiada que podría ayudar a ganar el caso. En esta situación, icuáles son las opciones del estudiante? En principio, debe poner en conocimiento del abogado supervisor el conflicto de intereses del cual es parte. De este modo, el abogado podrá tomar las medidas necesarias para lidiar con el dilema. Como obligación, debe notificarle al cliente lo acontecido, para que este pueda dar su consentimiento para que el estudiante siga viendo el caso; también deberá hacerlo respecto del tercero afectado — cuando se trate de un conflicto dispensableSi uno de ellos decide no consentir, entonces el abogado deberá impedir que el estudiante siga ayudando con el asunto encomendado; ante ello, al cliente se le asignará otro practicante, con la finalidad de que el primero pueda seguir adelante con su formación educativa y con su práctica.

¿Qué sucede, sin embargo, si es que el estudiante no se da cuenta del conflicto de intereses, en cualquiera de sus variantes? ¿Cabe asignarle al abogado la responsabilidad de identificarlo por el practicante?

Alexis Anderson, Arlene Kanter y Cindy Slane opinan, en el artículo ya citado, ${ }^{12}$ que el abogado supervisor deberá identificar y resolver, en nombre del practicante, la existencia de conflictos de intereses, ya que el alumno no cuenta con la proeza necesaria - pues no tiene experiencia en la asesoría legal - para hacerlo. El abogado será un soporte del practicante en este tipo de situaciones, y el alumno, a su vez, aprenderá a manejarlas, con lo cual se estarían cumpliendo los objetivos de formación profesional y de enseñanza que proveen las prácticas.

Otro supuesto sobre practicantes de Derecho en firmas de abogados se refiere al deber de competencia en relación con un asunto encomendado. Imaginémonos la siguiente situación: el abogado $\mathrm{Z}$ es supervisor

11 Anderson, Alexis, Arlene Kanter y Cindy Slane. «Ethics in Externships: Confidentiality, Conflicts and Competence Issues in the Field and in the Classrooms». Clinical Law Review, vol. 10, n 2, 2004, pp. 473-580. Base de datos Westlaw.

GRADUACIÓN

DE SANCIONES

EN LA RESPON-

SABILIDAD DEL ABOGADO POR

LAS FALTAS

COMETIDAS POR PERSONAS NO-

ABOGADAS QUE SE ENCUENTRAN BAJO SU SUPERVISIÓN 
del practicante $\mathrm{P}$, a quien asigna un asunto sobre un tema que este no conoce por no haberlo analizado en sus clases universitarias. El abogado $Z$, a la vez, conoce del tema asignado. En este caso no habría problema alguno, siempre y cuando se otorgue al alumno el tiempo necesario para realizar la investigación y el abogado efectivamente leyese el informe de su supervisado, lo corrigiera y señalase los aciertos y desaciertos sobre el tema. Así, el abogado estaría cumpliendo efectivamente el deber de enseñanza incluido como objetivo de las prácticas.

Pongámonos en otra variante del caso anterior. El practicante P investiga sobre el tema; sin embargo, por su complejidad, realiza un informe regular, que finalmente, si fuera presentado al cliente, no resolvería satisfactoriamente su consulta. El abogado Z, por la carga de trabajo que tiene, lee superficialmente el informe y se lo envía al cliente.

¿Qué tipo de responsabilidad tiene el abogado en este supuesto? Teniendo en cuenta que no está cumpliendo con el deber de enseñanza hacia el practicante - ya que no analiza su informe- - y que tampoco supervisa efectivamente su trabajo, si es que el cliente se queja por el informe deficiente enviado, el abogado no podría culpar al practicante por ello, ya que su obligación es corregir lo producido por este. Es decir, no podría trasladar la responsabilidad a la persona no-abogada que supervisa, aun así esta última hubiera incumplido con el deber de competencia exigido, ya que la delegación realizada no exime al abogado frente al cliente por la absolución de su consulta.

\section{Jurisprudencia}

Un primer supuesto que encontramos es el caso de aquel abogado que contrata a un investigador directamente y que le ordena contactar a un muchacho adolescente para ofrecerle una laptop a cambio de su computadora. ${ }^{13}$ El quid del caso era que el cliente del abogado era sospechoso de abuso de menores, con lo que el abogado estaba en realidad obteniendo del medio evidencia que podía ser utilizada por la fiscalía para formular una acusación contra su cliente. Acá, la falta cometida por el investigador devino de la orden directa del abogado, que tenía completo conocimiento de los alcances e implicancias de su decisión. Es evidente que el abogado debe responder completamente por la falta cometida, toda vez que no solo fue consciente de su decisión, sino que conocía que esta resultaría perjudicial para la contraparte en el caso - los menores que fueron víctimas del abuso del cliente- - y estaba obstruyendo también la investigación policial, al extraer evidencia del medio.

Un segundo supuesto que encontramos se presenta en el caso Nemecek vs. Karamacoski, en el que dos miembros del personal una firma legal no mandaron a tiempo la respuesta del abogado sobre una petición de

13 Caso de queja contra el abogado Stephen Hurley, febrero de 2008, Corte de Wisconsin. En TemkIn, Barry R. Op. cit., p. 1. 
la contraparte en un caso, lo cual ocasionó un perjuicio para su cliente. La Corte del Distrito Norte de Indiana declaró que este era un caso de negligencia excusable, ya que cada uno de los miembros se confundió y creyó razonablemente que el otro había enviado el respectivo documento. Sin embargo, consideró que solo bastaba que el abogado preguntara a cualquiera de ellos si es que habían realizado lo solicitado — o, en todo caso, que pidiese el cargo por el escrito enviado - para verificar que esto efectivamente se hubiera realizado. Este «mecanismo» de confirmación del deber del personal no tomaba mucho tiempo para el abogado — una simple llamada hubiera bastado- y hubiera podido evitar el perjuicio del cliente. En ese sentido, el comportamiento del abogado encargado fue negligente, máxime si tenía al alcance los medios para ser diligente.

\section{GRADUACIÓN DE SANCIONES}

En el presente capítulo, demostraremos la importancia de realizar la graduación de sanciones, los criterios utilizados para ello y, finalmente, presentaremos un cuadro con los supuestos estudiados anteriormente.

La graduación de sanciones se refiere a la gravedad, de grado mayor, medio o leve, que podemos encontrar en diversos supuestos de la vida profesional del abogado. Podremos identificar los valores vulnerados en cada situación y la responsabilidad asignada al abogado por las faltas cometidas por las personas no-abogadas que se encuentran bajo su supervisión. En ese sentido, es necesario establecer ciertos criterios para determinar la graduación de sanciones.

El primero que encontramos es un presupuesto necesario para la imputación de responsabilidad: que el abogado tenga efectivamente el deber de supervisión sobre la persona no-abogada que cometió la falta, o que tenga cierto control de manejo en el estudio legal. La primera acotación es fácil de identificar, ya que implica colaboración directa o labor directa con el abogado. La segunda acotación, sin embargo, genera muchas dudas. Surge el interrogante de si cualquier socio de un estudio jurídico tiene control de manejo o de si se debe delimitar el concepto. En teoría, todo socio tiene control de manejo de la firma de abogados — por la capacidad de toma de decisiones que implica, entre otras cosas-. Sin embargo, en una organización grande, es absurdo creer que el socio del área tributaria, por ejemplo, pueda ser responsable por una falta cometida por un investigador que realiza una labor para el área procesal. Tal como Robert R. Keatinge expone, la delimitación se refiere principalmente al control posible y efectivo que tiene el socio en el área a la que pertenece. ${ }^{14}$

GRADUACIÓN

DE SANCIONES

EN LA RESPON-

SABILIDAD DEL

ABOGADO POR

LAS FALTAS

COMETIDAS POR

PERSONAS NO-

ABOGADAS QUE

SE ENCUENTRAN

BAJO SU SUPER-

VISIÓN 
El segundo criterio que podemos aplicar es el de la determinación de si el abogado tenía conocimiento de la comisión de la falta; es decir, si es que el abogado ordenó a su supervisado la comisión de la falta, en cuyo caso tendría responsabilidad directa por ser parte de la realización de esta y de los efectos negativos derivados sobre terceros.

Dentro de este criterio, e independientemente del razonamiento anterior, también debemos analizar si, una vez conocida la falta — después de su comisión-, el abogado sancionó a la persona responsable o si, por el contrario, ratificó esta conducta indebida. Si se deja impune este tipo de comportamientos, se afecta, como vimos anteriormente, la apariencia debida de la profesión, lo cual es algo inaceptable.

El tercer criterio que debe ser considerado es el de la diligencia del abogado respecto del deber de supervisión que tiene dentro de la organización a la que pertenece. Como ya desarrollamos en el segundo capítulo, simplemente debemos resaltar que la diligencia tiene como límite la racionabilidad; es decir, el abogado tiene que monitorear razonablemente las actividades de las personas que se encuentran bajo su cargo, ya que, aunque delegue ciertas tareas, es en principio responsable por ellas y debe atender que le fueron encomendadas a él. Asimismo, además de la responsabilidad, se deben tomar en consideración los elementos de negligencia y de culpa en la actuación del abogado — respecto de la supervisión sobre las personas que se encuentran bajo su cargo-.

Un cuarto criterio que sirve para establecer la graduación de sanciones es el contenido del deber de supervisión. Este no solo implica el deber de monitoreo, sino que se puede encontrar en tres aspectos específicos, tal y como Robert R. Keatinge lo desarrolla en su ensayo. Estos son:

1. La supervisión directa, en la que el abogado tiene que instaurar razonablemente las medidas necesarias para que la persona noabogada se comporte de acuerdo con las reglas de la ética.

2. El deber de establecer mecanismos que aseguren el compromiso de las personas no-abogadas de actuar de acuerdo con las reglas de conducta profesional.

3. El deber de curar la vulneración derivada de la falta del supervisado. De este modo, se alienta a abogados y socios a mitigar los efectos negativos cuando aún están a tiempo de hacerlo.

Que se incumpla uno de estos tres aspectos del deber de supervisión implica la vulneración del deber mismo, con lo que el abogado supervisor incurriría en responsabilidad ética.

Un quinto criterio a ser considerado es el de la negligencia excusable, el cual ha sido ampliamente desarrollado por la jurisprudencia estadounidense. Se señala que existen ciertas situaciones que se encuentran fuera del alcance del abogado, respecto de las actuaciones de las personas que se encuentran bajo su supervisión. $\mathrm{O}$, simplemente, que 
las faltas cometidas por estas últimas no implican la responsabilidad del abogado por una serie de circunstancias que rodean el caso concreto. La jurisprudencia se basa fundamentalmente sobre cuatro factores que deben ser tomados en cuenta para determinar si se libera al abogado de su responsabilidad: el peligro del perjuicio al tercero (el afectado); la duración de la dilación y su impacto en los procesos judiciales; la razón de la dilación - y si había una posibilidad razonable de control sobre la falta-; y la buena fe del sujeto que cometió la infracción.

Finalmente, el último criterio a ser considerado es si es que se afectan otras normas éticas cuando se incurre en las faltas y la cantidad de reglas que se vulneran.

Presentamos un cuadro en el que se agrupan los supuestos de imputación y se les aplica la graduación de sanciones:

\begin{tabular}{|l|l|}
\hline $\begin{array}{l}\text { Supuestos graves de } \\
\text { sanción }\end{array}$ & $\begin{array}{l}\text { Cuando el abogado ordena directamente al supervisado la } \\
\text { comisión de la falta. } \\
\text { Cuando se tiene conocimiento de la comisión de la infrac- } \\
\text { ción y no se mitigan o evitan sus consecuencias. } \\
\text { Cuando el investigador se apropia de información relevan- } \\
\text { te para el caso. } \\
\text { Cuando cualquiera de las personas no-abogadas supervisa- } \\
\text { das incurre en una infracción del secreto profesional. }\end{array}$ \\
\hline $\begin{array}{l}\text { Supuestos moderados } \\
\text { de sanción }\end{array}$ & $\begin{array}{l}\text { Cuando el personal de la firma legal, por confusión, no en- } \\
\text { vía un escrito. } \\
\text { Cuando el investigador contacta a la contraparte y le son- } \\
\text { saca información del caso. } \\
\text { Cuando la firma legal falla en instaurar mecanismos gene- } \\
\text { rales que requieran que las personas no-abogadas se com- } \\
\text { porten de acuerdo con reglas de conducta profesional. } \\
\text { Cuando el abogado falla en supervisar el trabajo del prac- } \\
\text { ticante, sobre un tema que este último nunca ha visto, y se } \\
\text { lo envía al cliente. }\end{array}$ \\
\hline $\begin{array}{l}\text { Supuestos leves de } \\
\text { sanción }\end{array}$ & $\begin{array}{l}\text { Cuando el practicante no reconoce que incurre en conflicto } \\
\text { de intereses (en cualquiera de sus variantes dispensables). }\end{array}$ \\
\hline
\end{tabular}

GRADUACIÓN

DE SANCIONES

EN LA RESPON-

SABILIDAD DEL

ABOGADO POR

LAS FALTAS

COMETIDAS POR PERSONAS NO-

ABOGADAS QUE SE ENCUENTRAN BAJO SU SUPERVISIÓN

Los supuestos leves de sanción responden a una infracción leve de los deberes correspondientes en el caso concreto. El supuesto que se encuentra en este nivel existe porque, al no poder el practicante identificar siquiera el conflicto de intereses, el abogado no puede inferirlo razonablemente, máxime si es que no conoce todos los asuntos vistos por el primero.

Por otro lado, los supuestos moderados de sanción responden a faltas que atentan contra valores que guarda la profesión, pero que no los afectan completamente y que pudieron ser razonablemente evitados por 
el abogado, quien, por descuido, no lo hizo. En estos casos, sobre todo se afecta el deber de diligencia debida y el de una efectiva supervisión.

Finalmente, los supuestos graves de sanción se manifiestan en el marco de la vulneración grosera de los deberes fundamentales de la profesión, como el secreto profesional o la conducta debida del abogado. Así, la responsabilidad del abogado no se establece solo sobre la base de la falta cometida por la persona supervisada, sino también, en algunos casos, por su participación directa en la comisión de esta o por su flagrante negligencia.

\section{BIBLIOGRAFÍA COMPLEMENTARIA \\ Código Deontológico de la Comunidad Europea.}

MilLeR, Irwin, D. «Preventing Misconduct by Promoting the Ethics of Attorney's Supervisory Duties». Notre Dame Law Review, vol. 80, 1994.

Model Rules of Professional Conduct ABA.

O'Sullivan, Julie Rose. «Professional Discipline for Law Firms? A Response to Professor Schneyer's Proposal». Georgetown Journal of Legal Ethics, 16, 2002, pp. $1-90$.

Richmond, Douglas R. «Subordinate Lawyers and Insubordinate Duties». West Virginia Law Review, nำ 105, 2003.

Simpson-Wood, Taylor. «A Litmus Test for Pioneer: Ethical Considerations and the Delegation Situation». Journal of the Legal Profession, vol. 31, 2007, pp. 171-178. 